\title{
Is solid copper oxalate a spin chain or a mixture of entangled spin pairs?
}

\author{
Pavel Pokhilko ${ }^{a}$, Dmitry S. Bezrukov ${ }^{b, c}$, and Anna I. Krylov ${ }^{a}$ \\ ${ }^{a}$ Department of Chemistry, University of Southern California, Los Angeles, California 90089-0482 \\ ${ }^{b}$ Department of Chemistry, M.V. Lomonosov Moscow State University, Moscow 119991, Russia \\ ${ }^{c}$ Skolkovo Institute of Science and Technology, Skolkovo \\ Innovation Center, Nobel str. 3, Moscow 143026, Russia
}

\begin{abstract}
Macroscopic assemblies of interacting spins give rise to a broad spectrum of behaviors determined by the spatial arrangement of the magnetic sites and the electronic interactions between them. Compounds of copper (II), in which each copper carries spin $\frac{1}{2}$, exhibit a vast variety of physical properties. For antiferromagnetically coupled spin sites, there are two limiting scenarios: spin chains in which the spins can exhibit a long-range order or a mixture of dimers in which the spins within each pair are entangled but do not communicate with the spins from other dimers. In principle, the two types can be distinguished on the basis of experimental observations and modeling using empirically parameterized effective Hamiltonians, but in practice, ambiguity may persist for decades, as is the case for copper oxalate. Here we use high-level $a b$ initio calculations to establish the validity of the nearestsite Heisenberg model and to predict the interaction strength between the magnetic sites. The computed magnetic susceptibility provides an unambiguous interpretation of magnetic experiments performed throughout half a century, clearly supporting the infinite spin-chain behavior of solid copper oxalate.
\end{abstract}

\section{INTRODUCTION}

Strongly correlated materials exhibit unusual properties, which are exploited in emerging applications such as quantum information science and spintronics[1]. Physical properties depend on the spatial arrangement and the strengths of electronic couplings between the magnetic sites, as illustrated in Fig. 1. For example, chains of antiferromagnetically coupled spins exhibit long-range spin correlations and a zero spectral gap at zero magnetic field. In 


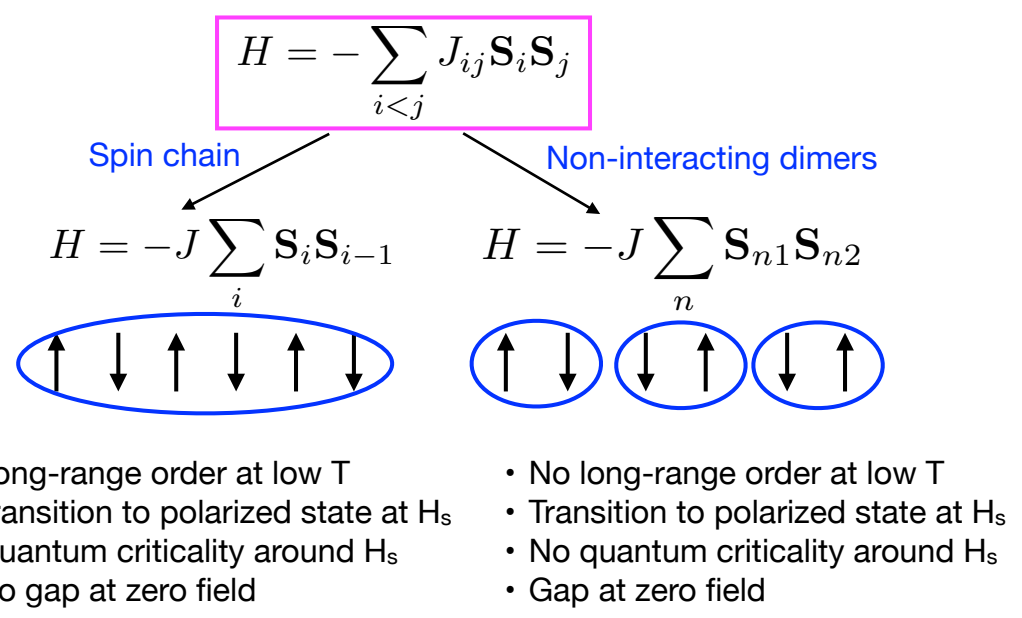

FIG. 1: An assembly of interacting spins is described by the Heisenberg Hamiltonian. Depending on the structure and strength of interactions, such system can exhibit vastly different physics. For one-dimensional systems, the two limiting cases are spin chains in which the spins can exhibit a long-range order or a mixture of dimers in which the spins within each pair are entangled but do not communicate with the spins from other dimers.

contrast, an assembly of weakly coupled spin pairs has no long-range order and has a gap at zero field. Both cases give rise to a bell-shape magnetic susceptibility curve $\chi(T)$. In both types of systems, the equilibrium state can be converted to a fully spin-polarized state above saturation field, roughly equal to the exchange coupling. The differences between the two cases can be seen at low temperatures, e.g., spin chains have finite $\chi(T)$, in contrast to dimers. The spectral gap and long-range order can also be probed by neutron scattering experiments, e.g., dynamic structural factors for the spin-chains at zero field start at zero energy and show characteristic sinusoidal shape, following the spinon dispersion relation $\epsilon(k)=\frac{\pi}{2}|J| \sin k$, as was illustrated for copper sulfate[2]; whereas the dimers are expected to show flat bands at finite energy. In addition to their technological relevance[1], spin chains are also fundamentally interesting - for example, they give rise to quantum criticality around the saturation field $[3,4]$.

Copper (II) salts can behave both as antiferromagnetic spin chains[2, 4] and as spinpaired dimers $[5,6]$. When experimental data is limited to finite-temperature magnetic susceptibility, as in the case of copper oxalate, one cannot confidently distinguish between the two regimes without first-principle calculations. Here we report the first fully ab initio determination of the effective Hamiltonian and macroscopic magnetic susceptibility for copper oxalate. The results provide unambiguous interpretation of magnetic experiments 
performed throughout half a century and clearly support the infinite spin chain behavior of the solid copper oxalate.

As shown in Fig. 1, an infinite chain of spins can be treated with the XXX Heisenberg Hamiltonian

$$
H=-J \sum_{i} \mathbf{S}_{i} \mathbf{S}_{i+1},
$$

where the $\mathbf{S}_{i}$ are local spins and $J$ is the effective exchange constant. If the effective exchange constant is negative, the system adopts the antiferromagnetic singlet ground state with opposite spin orientation of the adjacent magnetic centers. The case of $S=1 / 2$ is one of the few known quantum integrable models. Its exact solution is given by the Bethe ansatz[7], which facilitated the development of solid-state physics and mathematics of integrable systems. The thermodynamic properties of this model are well-known and have been used to explain experimental observables of real materials, containing, for instance, copper[4, 8], vanadium[9], and magnesium[10] magnetic centers.

Despite its simplicity, the magnetic structure of copper oxalate has not been settled. On the basis of susceptibility measurements, EPR, and EXAFS, both a dimer[11-13] and an infinite spin chain Heisenberg models[14, 15] have been proposed and used to fit the experimental data. Even after the determination of the crystal structure in 2014, the choice of magnetic model remained open[13].

Even when the model is known, the first-principle determination of the effective exchange constant is not trivial. The strongly correlated nature of magnetic systems combined with their large sizes, pose a formidable challenge for quantum chemistry. Here we use stateof-the-art wave-function methods based on equation-of-motion coupled-cluster (EOM-CC) theory[16]. In contrast to popular broken-symmetry density functional theory, our approach has no empirical or system-dependent parameters and does not rely on unphysical spinscrambled solutions. We begin with the full-electron treatment of model systems and use the resulting wave functions to build effective Hamiltonians[17, 18], which afford a coarsegrained description of the electronic structure and allow extrapolation to infinite systems. This is the first application of the spin-flip EOM-CC method to describe a periodic system, opening a new route in the treatment of periodic strongly correlated systems. Our theoretical 
results provide unambiguous interpretation of the magnetic measurements[11-15] of copper oxalate performed throughout several decades.

\section{RESULTS AND DISCUSSION}

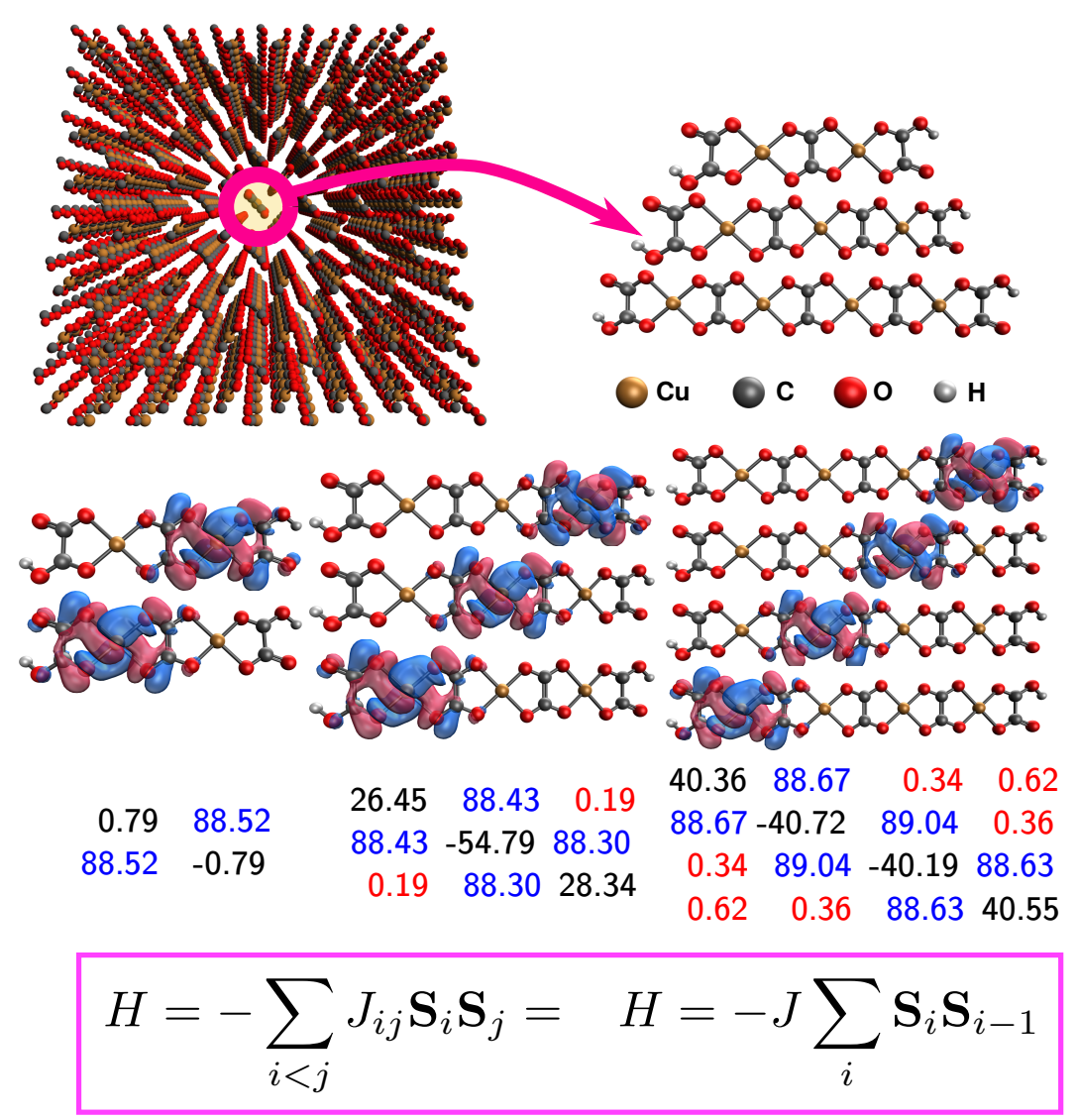

FIG. 2: Top, left: Crystal structure of copper oxalate $\left(\mathrm{CuC}_{2} \mathrm{O}_{4}\right)$. Top, right: fragments with 2, 3 , and 4 copper atoms from the crystal. Middle: Localized orbitals defining the open-shell model spaces. Bottom: Des Cloizeaux effective Hamiltonians built from the EOM-SF wave functions in the basis of open-shell determinants. The energies are shifted to produce a zero trace. The numbers in blue show the couplings between nearest neighbors. The numbers in red show the couplings between distant centers.

The crystal structure[13] of copper oxalate, shown in Figure 2, reveals a regular pattern of copper oxalate chains with a bidentate orientation of the oxalate ligands. We begin with EOM-SF-CCSD/cc-pVDZ calculations for the fragments of increasing length (containing 2, 3, and 4 copper centers, as shown in Figure 2, and capped with hydrogen atoms). We use the resulting energies and wave functions to build effective Hamiltonians[17, 18], which afford a coarse-grained description of the electronic structure. 
The effective Heisenberg Hamiltonians for fragments built from these highly accurate EOM-SF-CCSD calculations are shown in the middle panel of Figure 2. Bloch's and des Cloizeaux's versions of the effective Hamiltonians are consistent with each other and yield nearly the same values of effective exchange constants $J$, as shown in Table S2 in the SI. This procedure provides a rigorous mapping between the full-electron description and effective interactions between the magnetic centers. In particular, the resulting effective exchange constant includes not only the contribution from the exchange integral, but also screening and correlation effects, folded in by means of the Bloch wave operator. We then use the Heisenberg Hamiltonian to obtain the states that are not reachable by a single spin-flip, as was pioneered by Mayhall and Head-Gordon for single-molecule magnets[19].

Note that in these calculations we constructed the full Heisenberg Hamiltonian (as shown in the pink box in Fig. 1), i.e., without assuming nearest-neighbor approximation or constraining all neighboring $J$ to be the same. The analysis of these ab initio constructed effective Hamiltonians clearly reveals antiferromagnetic spin interactions. The off-diagonal elements decay rapidly with the distance between the copper centers, such that only the nearest-neighbor spin couplings are significant. Non-nearest-neighbor couplings are about $1 \mathrm{~cm}^{-1}$ or less, which is comparable with the thresholds used in the calculations. To further quantify the impact of neglecting the non-nearest neighbors contributions, we carried out two additional numeric tests. First, we computed eigenvalues of the Heisenberg Hamiltonian in which we zeroed the contributions from non-nearest neighbors and compared them with the eigenvalues of the full Hamiltonian; the results are shown in Table S3 of the SI. As one can see, the individual eigenvalues change by less than $0.5 \mathrm{~cm}^{-1}$ and the effect on the spectral gap is of the same magnitude - for example, the difference between the highest and the lowest eigenstate in the 4-copper Hamiltonian changes by $0.44 \mathrm{~cm}^{-1}$ due to the zeroing out non-nearest neighbor elements, which is indeed small compared to the value of the gap $\left(301.07 \mathrm{~cm}^{-1}\right)$. Second, we tri-diagonalized the full effective Hamiltonian for the 3 and 4 copper centers, so that the effect of non-nearest neighbor contributions is folded into the remaining matrix elements. The results, which are shown in Fig. S2 in the SI, show that this operation changes the value of the key matrix element (between the two middle coppers) by only $0.63 \mathrm{~cm}^{-1}$. Therefore, we conclude that the nearest-neighbor approximation is indeed fully justified for this system, such that the full Heisenberg Hamiltonian for the fragments can be replaced by the XXX Heisenberg Hamiltonian for the infinite spin chain, Eq. (1), 
which we use to extrapolate the Hamiltonian to the limit of the infinite number of copper centers. The effective exchange constants computed for the fragments of increasing sizes (collected in Table S2) show little variation and converge rapidly with respect to the model system size. Hence, for calculations of thermodynamic properties we use the $|J|$ constant from the middle of the 4-copper fragment $\left(-178.1 \mathrm{~cm}^{-1}\right)$.

To account for weak correlations beyond EOM-CCSD, we calculated a perturbative triples correction using the (fT) model for a model dimer. Surprisingly, inclusion of triple excitations leads to a more than $20 \%$ increase of $|J|\left(42.7 \mathrm{~cm}^{-1}\right)$, illustrating that weak correlations are important for quantitative results. The direction of the change can be explained by the Pauli repulsion principle. Pauli repulsion, which is already built-in in the wave-function at the mean-field level by virtue of using Slater determinants, allows the electrons in the triplet state to avoid each other. The resulting Pauli hole covers the errors due to an incomplete description of the Coulomb hole, which requires electron correlation. Consequently, the correlation effects are always smaller for triplet states[20] and improving correlation treatment results in stabilizing singlets relative to triplets. Previous calculations of dicopper singlemolecule magnets suggested that the cc-pVDZ basis is nearly sufficient[21, 22]. To account for a basis-set effect beyond cc-pVDZ, we performed EOM-SF-CCSD calculations for the dimer with the cc-pVTZ basis set. These calculations show that this improvement of the basis-set reduces the $|J|$ value by $8.5 \mathrm{~cm}^{-1}$.

To account for spin-orbit interaction, which can split and shift energy levels, we computed spin-orbit couplings (for the model dimer) using the Breit-Pauli Hamiltonian within spinorbit mean-field approximation and non-relativistic EOM-CC states. In these calculations we used the EOM-DIP-CCSD ansatz, which can describe not only the lowest singlet and triplet states (i.e., the Heisenberg states), but the entire manifold of the low-lying states derived from different occupations of coppers' $d$-orbitals. Although these states are relatively far in energy from the lowest singlet/triplet pair (about $1.6 \mathrm{eV}$ above the lowest singlet), they are expected to be important for spin-orbit coupling by virtue of El-Sayed's rule[2325], which says that states with different orbital occupancies give rise to large spin-orbit interactions.

The EOM-DIP-CCSD ansatz, which can handle strong correlation well but underestimates weak correlation and orbital relaxation, overestimates the energy gap relative to EOM-SF-CCSD $\left(J=-388.6 \mathrm{~cm}^{-1}\right.$ for the dimer). To correct for this limitation, we com- 
bined the EOM-SF-CCSD energy gap with EOM-DIP-CCSD spin-orbit couplings to evaluate the effective $|J|$. Table S4 illustrates the convergence of the $J$ constant with respect to the the lowest singlet number of interacting states. We observe that both the lowest singlet and the lowest triplet states interact strongly through the spin-orbit operator with higher excited states. This interaction shifts both states, largely canceling the effect of the spinorbit coupling on the singlet-triplet gap. Nevertheless, the singlet interacts more strongly with the excited states than the triplet does, which results in a substantial $(13 \%)$ increase of antiferromagnetic $|J|$ by $32 \mathrm{~cm}^{-1}$. We note that these results indicate that neglect of the spin-orbit interaction in previous studies of di-copper single-molecule magnets might be responsible for the reported systematic discrepancies between the theoretical and computed $J$-values[21].

Table I summarizes the various contributions to the effective $J$ and gives our best estimate of $J=-244 \mathrm{~cm}^{-1}=30.25 \mathrm{meV}$. Comparing to other copper spin chains, this value is 33 times higher than that of copper pyrazine[4] and 118 times higher than of copper sulfate[2], implying that a much stronger magnetic field (on the order of 400-500 T) would be required to bring this system to the fully polarized spin state and into the quantum criticality regime.

TABLE I: Contributions to the effective exchange constant $J, \mathrm{~cm}^{-1}$.

-178.1 Converged effective Hamiltonian

-42.7 Perturbative triples

-32 Spin-orbit shift

\begin{tabular}{ll}
+8.5 & Basis-set effects \\
\hline-244 & Total
\end{tabular}

To compare the computed effective exchange constants with the experimental observable, one needs to compute macroscopic magnetic susceptibility $\chi(T)$. Even with the solvable Bethe ansatz, such calculations require additional approximations. Here we consider two approaches: analytic classical and numeric quantum treatment of the temperature dependence of $\chi(T)$. In the classical treatment, the quantum Heisenberg Hamiltonian is mapped onto the classical Heisenberg Hamiltonian[26]. The connection between the quantum and classical parameters in the case of $S=1 / 2$ is given by

$$
\begin{gathered}
J^{\text {quant }} / 2=J^{c l}, \\
g^{\text {quant }}=g^{c l} .
\end{gathered}
$$


The analytic solution of the $\chi(T)$ exists for the case of a classical linear spin chain with nearest-neighbor approximation under the assumption of uniformness, meaning that $J_{i, i+1}$ and $g_{i}$ are the same for all local spins. The solution has the following form[26]:

$$
\begin{gathered}
\chi(T)=\frac{N\left(g^{c l}\right)^{2} \mu_{B}^{2}}{12 k T} \frac{1+u(K)}{1-u(K)}, \\
u(K)=\operatorname{coth} K-(1 / K), \\
K=\frac{J^{c l} / 2}{k T},
\end{gathered}
$$

where $N$ is the number of centers, $g^{c l}$ is a classical electron $g$-factor (we assumed $g^{c l}=$ $\left.g^{\text {quant }}=2\right), \mu_{B}$ is Bohr's magneton, and $k$ is the Boltzmann constant. This expression predicts a maximum on the susceptibility curve at:

$$
k T_{\max }^{\mathrm{cl}} \approx 0.2382\left|J^{c l}\right|
$$

The analytic expression for quantum susceptibility is not known, but there are Padé approximants[27] fitted to numerical curves with high quality. We used such a curve (Fit 1 in Table I in the Ref. [27]) for calculation of quantum magnetic susceptibility. The susceptibility curve of a quantum infinite spin chain has a maximum at

$$
k T_{\max } \approx 0.640851|J|
$$

One can immediately see that for the same value of $J^{\text {quant }}$, the classical model yields $\mathrm{T}_{\max }$, which is about 5.4 times lower that of the quantum model. Conversely, using the classical model to fit the experimental data would yield a 5.4 times larger effective exchange constant.

It is instructive to compare the predictions of the Heisenberg model with the dimer model, which gives the well-known Bleaney-Bowers expression for magnetic susceptibility[5]

$$
\chi^{\operatorname{dim}}(T)=\frac{N_{A} g^{2} \mu_{B}^{2}}{k T} \frac{2}{3+e^{-\frac{J}{k T}}}
$$

The susceptibility of the dimer reaches maximum at

$$
k T_{\text {max }}^{\operatorname{dim}} \approx 0.624|J| .
$$


Thus, although based on very different physics, the dimer model yields very similar value of $\mathrm{T}^{\max }$, i.e., within $3 \%$ from the quantum susceptibility derived from the Heisenberg model.
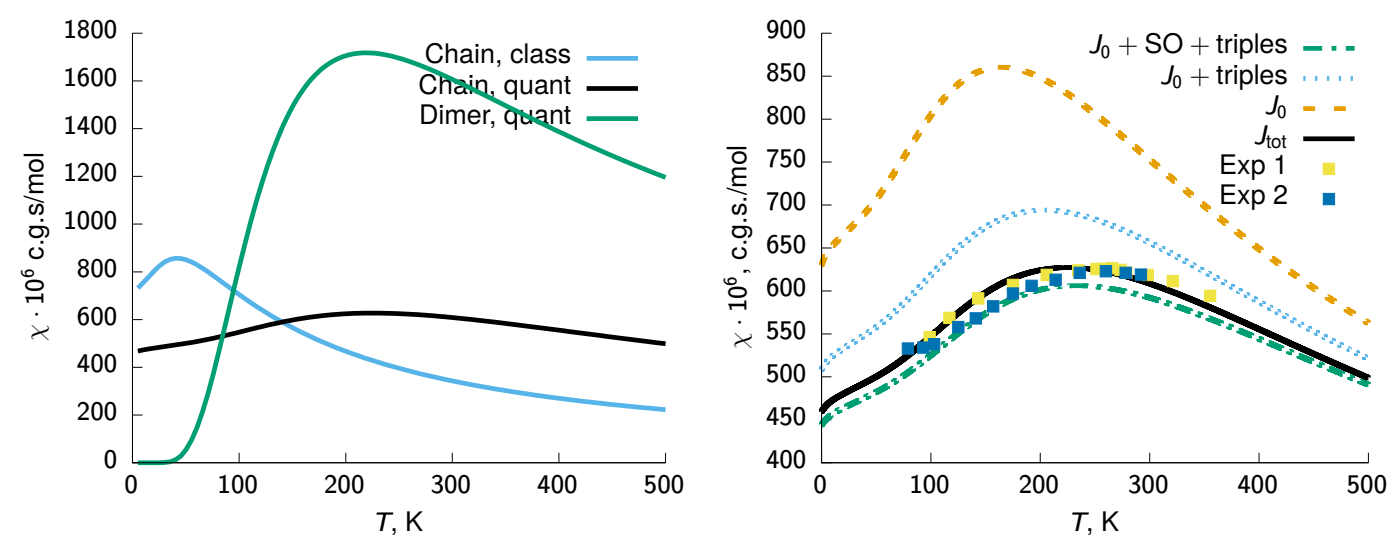

FIG. 3: Left: Classical and quantum susceptibilities of the XXX Heisenberg model and the susceptibility for a dimer model (Bleaney-Bowers expression) computed with $J=-244 \mathrm{~cm}^{-1}$. Right: Quantum susceptibility computed with the effective exchange constants from different approximations. $J_{0}$ is the converged value of the exchange coupling constant from the 4-center fragment. $J_{\text {tot }}=-244 \mathrm{~cm}^{-1}$ is the overall value of the coupling that includes $J_{0}$, perturbative triples, spinorbit shift, and basis-set extrapolation. The experimental data set 1 and 2 are from the Refs. [12] and [28], respectively.

The results of the classical and quantum Heisenberg models and the dimer model are shown in Fig. 3. The susceptibility of the classical spin model differs dramatically from the quantum spin model (Figure 3, left), making the classical approximation not satisfactory in the interpretation of the magnetic data. This is expected, since the susceptibility of the classical model approaches the susceptibility of the quantum model only for the large spin on each center[29]. Classical, quantum, and experimental susceptibility curves reach maximum at $42 \mathrm{~K}, 225 \mathrm{~K}$, and $260 \mathrm{~K}$, respectively. The quantum dimer model gives the maximum at $219 \mathrm{~K}$. The difference between the quantum infinite chain and the dimer Heisenberg models in terms of the maximum position of the $\chi(T)$ curve is not large, but the absolute values are vastly different (the dimer model yields absolute values that are about 4 times higher). In the SI (Fig. S4), we show the comparison between the quantum $\chi(T)$ and the scaled as well as scaled and shifted dimer's $\chi(T)$ curves. As one can see, if the dimer $\chi(T)$ is scaled to match the value of the quantum $\chi(T)$ at its maximum, then the difference in the slope at low and high temperatures is still clearly visible. One can scale and shifts dimer's $\chi(T)$ to match both the maximum and the high-energy part of the curve (as was done in Ref. [13]), but then the slope in the low-energy part remains visibly different. This analysis clearly 
shows that the infinite chain model reproduces not only the absolute value of $\chi(T)$ but also the overall shape of the curve much better than the scaled and shifted dimer model.

We also see that the $\chi(T)$ curve is broad and rather flat, which makes it difficult to precisely extract $T_{\max }$ from noisy experimental data. Hence, the comparison on the basis of the maximum may be misleading, and one should look at the overall shape. Near zero-temperature results would be important to further highlight the discrepancy, but, unfortunately, there are no reliable data, as the study that attempted such measurements clearly shows contamination of the sample[13].

The right panel of Figure 3 shows the impact of different effects on the overall magnetic susceptibility of the quantum spin chain model. The value of the effective exchange constant from the 4-center fragment results in a susceptibility curve that is far from the experimental one. Inclusion of spin-orbit effects and weak correlation through perturbative triples improves agreement. The impact of the basis set is relatively small but it improves agreement even further. The more effects that are included, the closer the agreement with the experiment becomes.

Inelastic neutron scattering allows one to probe the manifold of spin states of a magnetic system. The intensity of inelastic neutron scattering is proportional to the dynamic structure factor, given by

$$
S^{\sigma \sigma^{\prime}}(k, \omega)=\sum_{i}\left\langle\Psi_{G S}\left|S_{k}^{\sigma}\right| \Psi_{i}\right\rangle\left\langle\Psi_{i}\left|S_{k}^{\sigma^{\prime}}\right| \Psi_{G S}\right\rangle \mid \delta\left(\omega-\omega_{i}\right)
$$

where $S_{k}^{\sigma}=\frac{1}{\sqrt{N}} \sum_{j}^{N} e^{i q j} S_{j}^{\sigma}$ are the spin operators in the periodic representation, $\Psi_{G S}$ and $\Psi_{i}$ are the ground and excited states, and $\omega_{i}$ is the excitation energy of excited state $\Psi_{i}$. Mixtures of weakly interacting antiferromagnetic dimers would have a sharp intensity peak at the transfer energy matching the singlet-triplet gap[30, 31]. The energy position of the peak does not depend on the momentum transfer $k$. In contrast, extended spin chains show a characteristic dispersion law, where the intensity is large at the distribution of energies and wave-vectors[32, 33]. Thus, future neutron scattering experiments for copper oxalate could further confirm the infinite spin-chain model and tighten the experimental error bar on the effective exchange constant.

The rigorously derived $a b$ initio effective Hamiltonian for copper oxalate corresponds to the infinite Heisenberg spin chain model and validates the nearest-neighbor approximation. 
This is the first purely theoretical validation of the XXX Heisenberg model that is based on non-empirical all-electron many-body calculations and not relying on spin-symmetry broken solutions. Our results show that both strong and weak correlation contribute to the effective exchange constant, whereas the dependence on the basis set is relatively weak. The spin-orbit interaction introduces a noticeable additional shift that further changes the effective exchange constant. Only after accounting for all these effects, does the magnetic susceptibility of the quantum spin model agree well with the experimental values[12, 28]. This is the first application of the EOM-SF-CC method to describe a periodic system, opening a new route in the treatment of strongly correlated periodic systems. These results provide a solid basis for future quantitative modeling of spin chains, including magnetic field effects.

\section{METHODS}

Cartesian coordinates were extracted from the crystal unit cell parameters of copper oxalate $\left(\mathrm{CuC}_{2} \mathrm{O}_{4}\right)$ reported in Ref. 13. The crystal structure is composed of the polymer $\mathrm{CuC}_{2} \mathrm{O}_{4}$ (Figure 2). The unit cell was repeated and the $\mathrm{CuC}_{2} \mathrm{O}_{4}$ stripes were extracted. Hydrogen atoms were added to the oxygen ends of the chain with the corresponding to oxalic acid bond lengths and angles. The Cartesian coordinates for all structures are given in the SI.

All calculations were performed with the Q-Chem quantum chemistry package[34, 35]. We used Dunning's cc-pVDZ and cc-pVTZ basis sets for all atoms[36-39]. Unrestricted orbitals were used in calculations with open-shell references. To accelerate electronic structure calculations, we used single precision[40] for CCSD, intermediates, and EOM-SF-CCSD, combined with the libxm library for tensor contractions[41].

We used the open-shell frozen natural orbital truncation of the virtual space[22] with the truncation threshold corresponding to $99 \%$ of the total preserved occupation in the virtual space. Core electrons were frozen in all calculations. Triples corrections were computed using (fT) perturbative triples[42] for EOM-SF-CCSD. In the (fT) calculations for a model dimer, the 40 lowest occupied orbitals were frozen for the dimer fragment.

Calculations of spin-orbit couplings[43] were performed using EOM-DIP-CCSD wave functions[44]. 
Bloch's and des Cloizeaux's effective Hamiltonians were built for the open-shell model spaces of the determinants expressed in the open-shell localized orbitals following the procedure from Ref. [18]. Localized orbitals were determined using the Foster-Boys criterion of localization[45].

\section{Acknowledgments}

We are grateful to Professor Devin Matthews from the South Methodist University for his generous help with testing the effects of correlation beyond perturbative triples correction. This work is supported by the Department of Energy through the DE-SC0018910 grant.

P.P. current address is: Department of Chemistry, University of Michigan, Ann Arbor, Michigan 48109, USA

The authors declare the following competing financial interest(s): A.I.K. is a member of the Board of Directors and a part-owner of Q-Chem, Inc. 
[1] T. Jungwirth, X. Marti, P. Wadley, and J. Wunderlich, Antiferromagnetic spintronics, Nat. Nanotechnol. 11, 231 (2016).

[2] M. Mourigal, M. Enderle, A. Klöpperpieper, J.-S. Caux, A. Stunault, and H. M. Rønnow, Fractional spinon excitations in the quantum Heisenberg antiferromagnetic chain, Nat. Phys. 9, 435 (2013).

[3] P. Coleman and A. J. Schofield, Quantum criticality, Nature 433, 226 (2005).

[4] O. Breunig, M. Garst, A. Klümper, J. Rohrkamp, M. M. Turnbull, and T. Lorenz, Quantum criticality in the spin-1/2 Heisenberg chain system copper pyrazine dinitrate, Science 3 (2017).

[5] B. Bleaney and K. D. Bowers, Anomalous paramagnetism of copper acetate, Proc. R. Soc. London A 214, 451 (1952).

[6] R. Maurice, K. Sivalingam, D. Ganyushin, N. Guihéry, C. de Graaf, and F. Neese, Theoretical determination of the zero-field splitting in copper acetate monohydrate, Inorg. Chem. 50, 6229 (2011).

[7] H. Bethe, Zur Theorie der Metalle, Z. Phys. 71, 205 (1931).

[8] N. Motoyama, H. Eisaki, and S. Uchida, Magnetic susceptibility of ideal spin 1/2 Heisenberg antiferromagnetic chain systems, $\mathrm{Sr}_{2} \mathrm{CuO}_{3}$ and $\mathrm{SrCuO}_{2}$, Phys. Rev. Lett. 76, 3212 (1996).

[9] D. C. Johnston, J. W. Johnson, D. P. Goshorn, and A. J. Jacobson, Magnetic susceptibility of $(\mathrm{VO})_{2} \mathrm{P}_{2} \mathrm{O}_{7}$ : A one-dimensional spin-1/2 Heisenberg antiferromagnet with a ladder spin configuration and a singlet ground state, Phys. Rev. B 35, 219 (1987).

[10] P. M. Richards, R. K. Quinn, and B. Morosin, Magnetic and structural properties of manganese pyridine linear chain salts, J. Chem. Phys. 59, 4474 (1973).

[11] B. N. Figgis and D. J. Martin, The magnetic properties and structure of the cupric $\alpha, \omega-$ dicarboxylates, Inorg. Chem. 5, 100 (1966).

[12] L. Dubicki, C. M. Harris, E. Kokot, and R. L. Martin, Magnetic studies with copper (II) salts. VII. The structure of copper (II) $\alpha, \omega$-dicarboxylates and their amine derivatives, Inorg. Chem. 5, 93 (1966).

[13] A. N. Christensen, B. Lebech, N. H. Andersen, and J.-C. Grivel, The crystal structure of paramagnetic copper(II) oxalate $\left(\mathrm{CuC}_{2} \mathrm{O}_{4}\right)$ : formation and thermal decomposition of randomly stacked anisotropic nano-sized crystallites, Dalton Trans. 43, 16754 (2014). 
[14] T. Kenneth K. T. McGregor and Z. G. Soos, Anisotropic exchange and temperature-dependent electron paramagnetic resonance line width in one-dimensional copper(II) complexes. 2. Magnetic properties of copper(II) oxalate-1/3-water, Inorg. Chem. 15, 2159 (1976).

[15] A. Michalowicz, J. J. Girerd, and J. Goulon, EXAFS [extended x-ray absorption fine structure] determination of the copper oxalate structure. Relation between structure and magnetic properties, Inorg. Chem. 18, 3004 (1979).

[16] A. I. Krylov, Equation-of-motion coupled-cluster methods for open-shell and electronically excited species: The hitchhiker's guide to Fock space, Annu. Rev. Phys. Chem. 59, 433 (2008).

[17] J. P. Malrieu, R. Caballol, C. J. Calzado, C. de Graaf, and N. Guihéry, Magnetic interactions in molecules and highly correlated materials: Physical content, analytical derivation, and rigorous extraction of magnetic Hamiltonians, Chem. Rev. 114, 429 (2013).

[18] P. Pokhilko and A. I. Krylov, Effective Hamiltonians derived from equation-of-motion coupledcluster wave-functions: Theory and application to the Hubbard and Heisenberg Hamiltonians, J. Chem. Phys. 152, 094108 (2020).

[19] N. J. Mayhall and M. Head-Gordon, Computational quantum chemistry for multiple-site Heisenberg spin couplings made simple: Still only one spin-flip required, J. Phys. Chem. Lett. 6, $1982(2015)$.

[20] A. I. Krylov, Size-consistent wave functions for bond-breaking: The equation-of-motion spinflip model, Chem. Phys. Lett. 338, 375 (2001).

[21] N. Orms and A. I. Krylov, Singlet-triplet energy gaps and the degree of diradical character in binuclear copper molecular magnets characterized by spin-flip density functional theory, Phys. Chem. Chem. Phys. 20, 13127 (2018).

[22] P. Pokhilko, D. Izmodenov, and A. I. Krylov, Extension of frozen natural orbital approximation to open-shell references: Theory, implementation, and application to single-molecule magnets, J. Chem. Phys. 152, 034105 (2020).

[23] M. A. El-Sayed, Spin-orbit coupling and the radiationless processes in nitrogen heterocycles, J. Chem. Phys. 38, 2834 (1963).

[24] M. A. El-Sayed, Triplet state: Its radiative and non-radiative properties, Acc. Chem. Res. 1, 8 (1968).

[25] P. Pokhilko and A. I. Krylov, Quantitative El-Sayed rules for many-body wavefunctions from spinless transition density matrices, J. Phys. Chem. Lett. 10, 4857 (2019). 
[26] M. E. Fisher, Magnetism in one-dimensional systems - the Heisenberg model for infinite spin, Am. J. Phys. 32, 343 (1964).

[27] D. C. Johnston, R. K. Kremer, M. Troyer, X. Wang, A. Klümper, S. L. Bud'ko, A. F. Panchula, and P. C. Canfield, Thermodynamics of spin $S=1 / 2$ antiferromagnetic uniform and alternating-exchange Heisenberg chains, Phys. Rev. B 61, 9558 (2000).

[28] V. V. Zelentsov and T. G. Aminov, Magnetic susceptibility of cupric oxalate and cupric succinate, Dokl. Akad. Nauk SSSR 158, 1393 (1964).

[29] T. Xiang, Thermodynamics of quantum Heisenberg spin chains, Phys. Rev. B 58, 9142 (1998).

[30] A. Furrer and H. U. Güdel, Interference effects in neutron scattering from magnetic clusters, Phys. Rev. Lett. 39, 657 (1977).

[31] A. Zheludev, G. Shirane, Y. Sasago, M. Hase, and K. Uchinokura, Dimerized ground state and magnetic excitations in $\mathrm{CaCuGe}_{2} \mathrm{O}_{6}$, Phys. Rev. B 53, 11642 (1996).

[32] J.-S. Caux, R. Hagemans, and J. M. Maillet, Computation of dynamical correlation functions of Heisenberg chains: the gapless anisotropic regime, J. Stat. Mech. Theory Exp 2005, P09003 (2005).

[33] M. Kohno, Dynamically dominant excitations of string solutions in the spin- $1 / 2$ antiferromagnetic Heisenberg chain in a magnetic field, Phys. Rev. Lett. 102, 037203 (2009).

[34] Y. Shao, Z. Gan, E. Epifanovsky, A.T.B. Gilbert, M. Wormit, J. Kussmann, A.W. Lange, A. Behn, J. Deng, X. Feng, D. Ghosh, M. Goldey, P.R. Horn, L.D. Jacobson, I. Kaliman, R.Z. Khaliullin, T. Kus, A. Landau, J. Liu, E.I. Proynov, Y.M. Rhee, R.M. Richard, M.A. Rohrdanz, R.P. Steele, E.J. Sundstrom, H.L. Woodcock III, P.M. Zimmerman, D. Zuev, B. Albrecht, E. Alguires, B. Austin, G.J.O. Beran, Y.A. Bernard, E. Berquist, K. Brandhorst, K.B. Bravaya, S.T. Brown, D. Casanova, C.-M. Chang, Y. Chen, S.H. Chien, K.D. Closser, D.L. Crittenden, M. Diedenhofen, R.A. DiStasio Jr., H. Do, A.D. Dutoi, R.G. Edgar, S. Fatehi, L. Fusti-Molnar, A. Ghysels, A. Golubeva-Zadorozhnaya, J. Gomes, M.W.D. HansonHeine, P.H.P. Harbach, A.W. Hauser, E.G. Hohenstein, Z.C. Holden, T.-C. Jagau, H. Ji, B. Kaduk, K. Khistyaev, J. Kim, J. Kim, R.A. King, P. Klunzinger, D. Kosenkov, T. Kowalczyk, C.M. Krauter, K.U. Laog, A. Laurent, K.V. Lawler, S.V. Levchenko, C.Y. Lin, F. Liu, E. Livshits, R.C. Lochan, A. Luenser, P. Manohar, S.F. Manzer, S.-P. Mao, N. Mardirossian, A.V. Marenich, S.A. Maurer, N.J. Mayhall, C.M. Oana, R. Olivares-Amaya, D.P. O’Neill, J.A. Parkhill, T.M. Perrine, R. Peverati, P.A. Pieniazek, A. Prociuk, D.R. Rehn, E. Rosta, 
N.J. Russ, N. Sergueev, S.M. Sharada, S. Sharmaa, D.W. Small, A. Sodt, T. Stein, D. Stuck, Y.-C. Su, A.J.W. Thom, T. Tsuchimochi, L. Vogt, O. Vydrov, T. Wang, M.A. Watson, J. Wenzel, A. White, C.F. Williams, V. Vanovschi, S. Yeganeh, S.R. Yost, Z.-Q. You, I.Y. Zhang, X. Zhang, Y. Zhou, B.R. Brooks, G.K.L. Chan, D.M. Chipman, C.J. Cramer, W.A. Goddard III, M.S. Gordon, W.J. Hehre, A. Klamt, H.F. Schaefer III, M.W. Schmidt, C.D. Sherrill, D.G. Truhlar, A. Warshel, X. Xu, A. Aspuru-Guzik, R. Baer, A.T. Bell, N.A. Besley, J.-D. Chai, A. Dreuw, B.D. Dunietz, T.R. Furlani, S.R. Gwaltney, C.-P. Hsu, Y. Jung, J. Kong, D.S. Lambrecht, W.Z. Liang, C. Ochsenfeld, V.A. Rassolov, L.V. Slipchenko, J.E. Subotnik, T. Van Voorhis, J.M. Herbert, A.I. Krylov, P.M.W. Gill, and M. Head-Gordon, Advances in molecular quantum chemistry contained in the Q-Chem 4 program package, Mol. Phys. 113, $184(2015)$.

[35] A. I. Krylov and P. M. W. Gill, Q-Chem: An engine for innovation, WIREs: Comput. Mol. Sci. 3, 317 (2013).

[36] T. H. Dunning, Jr., Gaussian basis sets for use in correlated molecular calculations. I. The atoms boron through neon and hydrogen, J. Chem. Phys. 90, 1007 (1989).

[37] N. B. Balabanov and K. A. Peterson, Systematically convergent basis sets for transition metals. I. All-electron correlation consistent basis sets for the 3d elements Sc-Zn, J. Chem. Phys. 123, 064107 (2005).

[38] J. Koput and K. A. Peterson, Ab initio potential energy surface and vibrational-rotational energy levels of $\mathrm{X}^{2} \Sigma^{+} \mathrm{CaOH}$, J. Phys. Chem. A 106, 9595 (2002).

[39] A. K. Wilson, D. E. Woon, K. A. Peterson, and T. H. Dunning, Gaussian basis sets for use in correlated molecular calculations. ix. the atoms gallium through krypton, J. Chem. Phys. 110, 7667 (1999).

[40] P. Pokhilko, E. Epifanovskii, and A. I. Krylov, Double precision is not needed for many-body calculations: Emergent conventional wisdom, J. Chem. Theory Comput. 14, 4088 (2018).

[41] I. Kaliman and A. I. Krylov, New algorithm for tensor contractions on multi-core CPUs, GPUs, and accelerators enables CCSD and EOM-CCSD calculations with over 1000 basis functions on a single compute node, J. Comput. Chem. 38, 842 (2017).

[42] P. U. Manohar and A. I. Krylov, A non-iterative perturbative triples correction for the spin-flipping and spin-conserving equation-of-motion coupled-cluster methods with single and double substitutions, J. Chem. Phys. 129, 194105 (2008). 
[43] P. Pokhilko, E. Epifanovsky, and A. I. Krylov, General framework for calculating spin-orbit couplings using spinless one-particle density matrices: theory and application to the equationof-motion coupled-cluster wave functions, J. Chem. Phys. 151, 034106 (2019).

[44] H. Zhao, Z. Wang, M. Guo, and F. Wang, Splittings of d8 configurations of late-transition metals with eom-dip-ccsd and fsccsd methods, J. Chem. Phys. 152, 134105 (2020).

[45] S. F. Boys, Construction of some molecular orbitals to be approximately invariant for changes from one molecule to another, Rev. Mod. Phys. 32, 296 (1960). 


\title{
Is solid copper oxalate a spin chain or a mixture of entangled spin pairs? Supplemental Information.
}

\author{
Pavel Pokhilko ${ }^{a}$, Dmitry S. Bezrukov ${ }^{b, c}$, and Anna I. Krylov ${ }^{a}$ \\ ${ }^{a}$ Department of Chemistry, University of Southern California, Los Angeles, California 90089-0482 \\ ${ }^{b}$ Department of Chemistry, M.V. Lomonosov Moscow State University, Moscow 119991, Russia \\ ${ }^{c}$ Skolkovo Institute of Science and Technology, Skolkovo Innovation Center, Nobel str. 3, Moscow 143026, Russia
}

December 16, 2020

\section{Effective Hamiltonians}
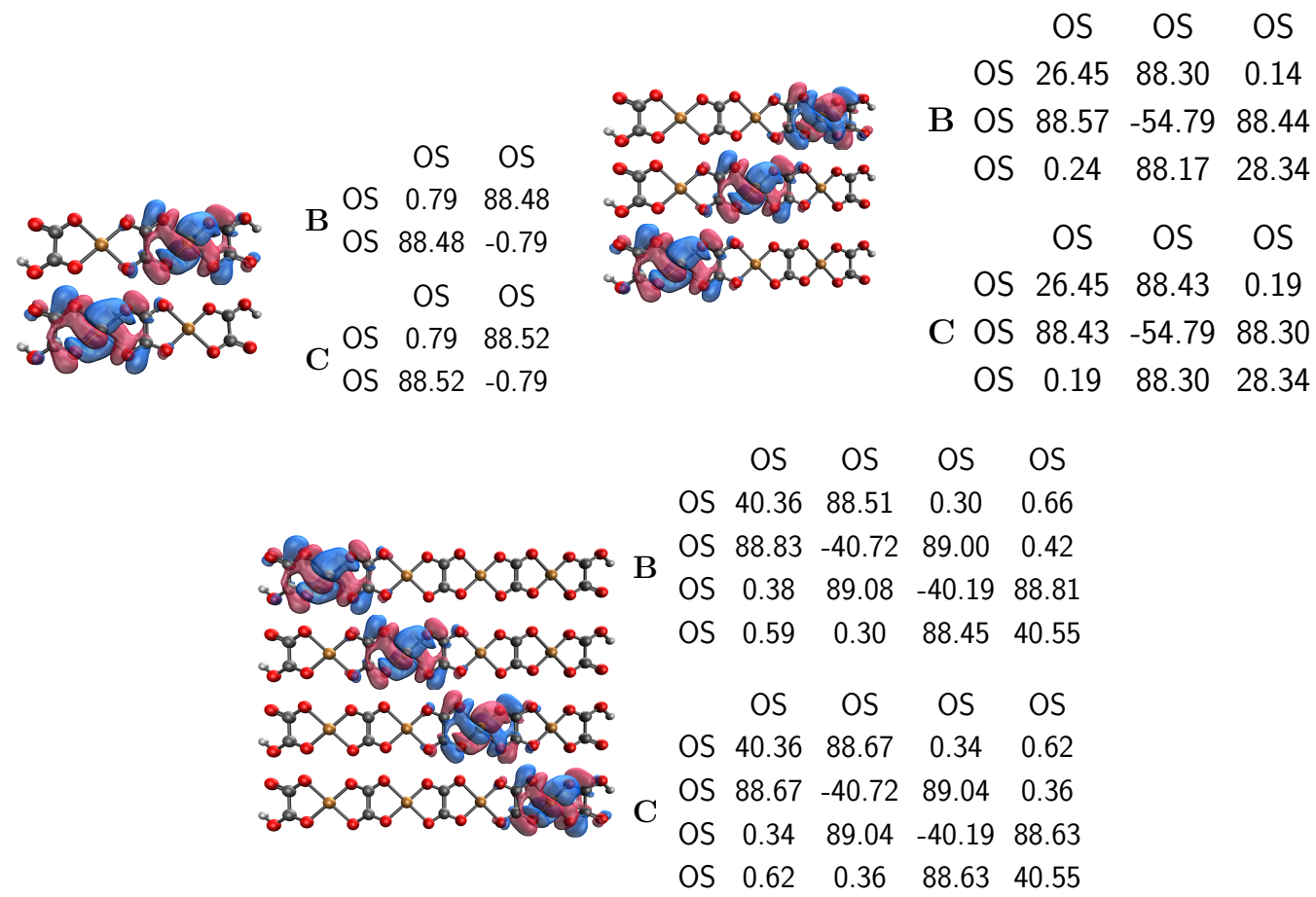

Figure S1: Bloch's (B) and des Cloizeaux's (C) effective Hamiltonians for the bi- and trinuclear fragment. The energies are shifted to produce a zero trace. 
Table S1: $\left\langle S^{2}\right\rangle$ of the reference determinants used in calculations and a comparison with spinpure solutions.

\begin{tabular}{l|c|c|c}
\hline$\#$ of $\mathrm{Cu}$ & 2 & 3 & 4 \\
\hline Spin-pure solution & 2.00 & 3.75 & 6.00 \\
\hline UHF & 2.007 & 3.760 & 6.013 \\
\hline
\end{tabular}

Table S2: Effective exchange couplings $J$ extracted with EOM-SF-CCSD/cc-pVDZ. Bloch's and des Cloizeax's effective Hamiltonians provide nearly identical values of $J$.

\begin{tabular}{l|c|c}
\hline$\#$ of $\mathrm{Cu}$ & Bloch & Des Cloizeaux \\
\hline 2 & -176.96 & -177.04 \\
3 & $-176.87,-176.61,-0.38$ & $-176.86,-176.60,-0.38$ \\
4 & $-177.34,-178.08,-177.26,-0.68,-0.72,-1.25$ & $-177.34,-178.08,-177.26,-0.68,-0.72,-1.24$ \\
\hline
\end{tabular}

Table S3: Impact of truncation to the nearest neighbors on the eigenvalues of the des Cloizeaux effective Hamiltonian. Eigenvalues (in $\mathrm{cm}^{-1}$ ) for the full and the truncated Hamiltonians are shown.

\begin{tabular}{l|c|c}
\hline$\#$ of $\mathrm{Cu}$ & Full & Nearest-neighbor truncation \\
\hline 3 & $-145.18,27.20,117.98$ & $-145.25,27.39,117.86$ \\
4 & $-167.16,-44.25,77.50,133.91$ & $-167.19,-44.40,78.15,133.44$ \\
\hline
\end{tabular}

$\begin{array}{rrrrrrrr}26.45 & 88.43 & 0.19 & 40.36 & 88.67 & 0.34 & 0.62 \\ 88.43 & -54.79 & 88.30 & 88.67 & -40.72 & 89.04 & 0.36 \\ 0.19 & 88.30 & 28.34 & 0.34 & 89.04 & -40.19 & 88.63 \\ & & & 0.62 & 0.36 & 88.63 & 40.55 \\ & \downarrow & & & & & \\ & \downarrow & & & & & \\ 26.45 & 88.43 & 0.00 & 40.36 & 88.67 & 0.00 & 0.00 \\ 88.43 & -54.41 & 88.48 & 88.67 & -40.02 & 89.67 & 0.00 \\ 0.00 & 88.48 & 27.96 & & 0.00 & 89.67 & -38.38 & 89.12 \\ & & & 0.00 & 0.00 & 89.12 & 38.04\end{array}$

Figure S2: Tridiagonalization of the des Cloizeaux $(\mathbf{C})$ effective Hamiltonians for the tri- and tetranuclear fragment. 


\section{Spin-orbit calculations}

As we noted in the main text, EOM-DIP-CCSD suffers from insufficient treatment of weak correlation and orbital relaxation. However, the energy shift due to spin-orbit interaction does not depend much on the value of the triplet-singlet gap (we report the results with the EOMSF-CCSD gap in the Table S4). This can be rationalized from El-Sayed's rule: the Heisenberg states have nearly the same orbital occupancies; therefore, there is no contribution from the direct coupling between them. The relative energy gaps with the other excited states are much larger, which makes the spin-orbit shifts insensitive to perturbations in the triplet-singlet gap of hundreds of $\mathrm{cm}^{-1}$.

Table S4: Positions $\left(\mathrm{cm}^{-1}\right)$ of the lowest singlet and triplet states after diagonalization of the spin-orbit Hamiltonian of the binuclear fragment. Three components of the triplet states are shown. The last column shows the effect of the spin-orbit couplings on the effective exchange constant. The zero energy corresponds to the energy of the singlet state prior incorporation of spin-orbit effects. The positions of the triplet states has been shifted by the same value prior diagonalization of the spin-orbit Hamiltonians to reproduce EOM-SF-CCSD triplet-singlet gap $\left(177 \mathrm{~cm}^{-1}\right)$.

\begin{tabular}{l|cccc|c}
\hline \# of states & $\mathrm{S}$ & $\mathrm{T}$ & $\mathrm{T}$ & $\mathrm{T}$ & $\Delta J$ \\
$2 \mathrm{~S}, 2 \mathrm{~T}$ & -65.3 & 177 & 177 & 177 & -65.3 \\
$3 \mathrm{~S}, 3 \mathrm{~T}$ & -65.4 & 109.6 & 109.6 & 177 & -20.5 \\
$4 \mathrm{~S}, 4 \mathrm{~T}$ & -75.0 & 109.5 & 109.6 & 177 & -30.0 \\
$5 \mathrm{~S}, 5 \mathrm{~T}$ & -75.0 & 98.7 & 109.6 & 165.0 & -22.4 \\
$6 \mathrm{~S}, 6 \mathrm{~T}$ & -75.1 & 95.1 & 100.3 & 152.6 & -14.1 \\
$7 \mathrm{~S}, 7 \mathrm{~T}$ & -89.2 & 95.0 & 100.3 & 152.5 & -28.1 \\
$8 \mathrm{~S}, 8 \mathrm{~T}$ & -90.1 & 86.7 & 92.1 & 146.1 & -21.4 \\
$9 \mathrm{~S}, 9 \mathrm{~T}$ & -100.1 & 85.7 & 91.5 & 145.5 & -30.7 \\
$10 \mathrm{~S}, 10 \mathrm{~T}$ & -100.9 & 85.7 & 91.5 & 145.5 & -31.5 \\
\hline
\end{tabular}




\section{Relationship between classical and quantum Heisen- berg models}

$$
\begin{gathered}
H^{c l}=-\frac{1}{2} \sum_{i<j} J_{i j}^{c l} \mathbf{s}_{i}^{c l} \cdot \mathbf{s}_{j}^{c l}-\frac{1}{2} \sum_{i} g_{i}^{c l} \beta \mathbf{H} \cdot \mathbf{s}_{i}, \\
\mathbf{s}^{c l}=\frac{\mathbf{S}^{c l}}{S}
\end{gathered}
$$

where the index $c l$ denotes a classical quantity. The correspondence between quantum quantities and their classical analogies is established through the following relations:

$$
\begin{aligned}
J^{\text {quant }} S^{2} & =\frac{1}{2} J^{c l}, \\
g^{\text {quant }} S & =\frac{1}{2} g^{c l} .
\end{aligned}
$$

For the convenience of the reader, we use consistent definition of the Heisenberg Hamiltonian and exchange constants. If comparison with other texts shall be made, one should compare definitions of the Hamiltonians. For example, we use $H=-\sum_{i<j} J_{i j}^{\text {our,quant }} S_{i} S_{j}=$ $-\frac{1}{2} \sum_{i \neq j} J_{i j}^{\text {our,quant }} S_{i} S_{j}$. Fisher used $H=-\sum_{i \neq j} J_{i j}^{\text {Fisher,quant }} S_{i} S_{j}$. Therefore, $J^{\text {Fisher,quant }}=$ $J^{\text {our, quant }} / 2$.

\section{Comparison with experiment}

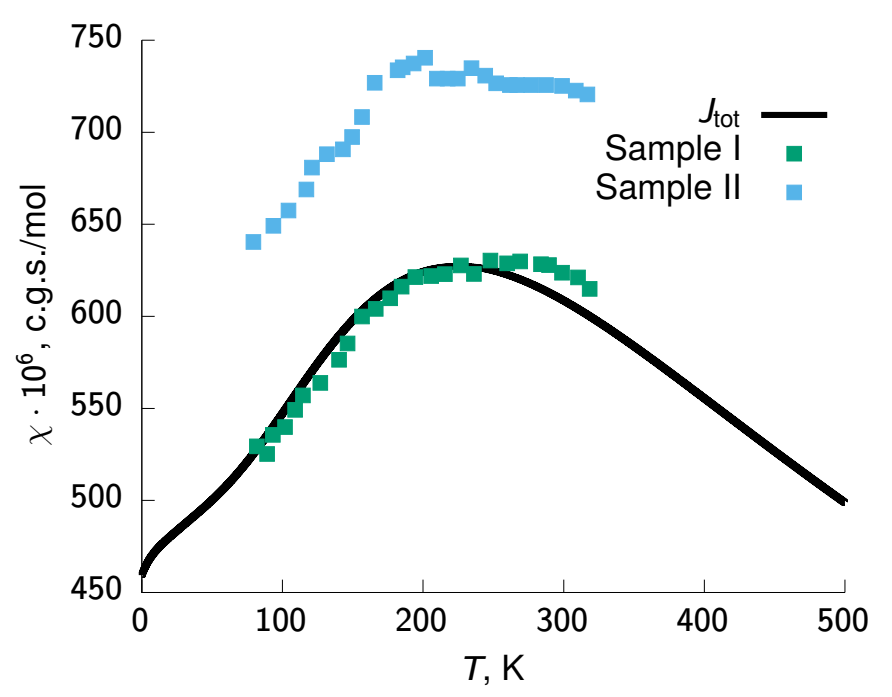

Figure S3: Comparison between our best theoretical estimate $\left(J_{\text {tot }}=244 \mathrm{~cm}^{-1}\right)$ and experimental measurements of two samples from the Ref.1. 


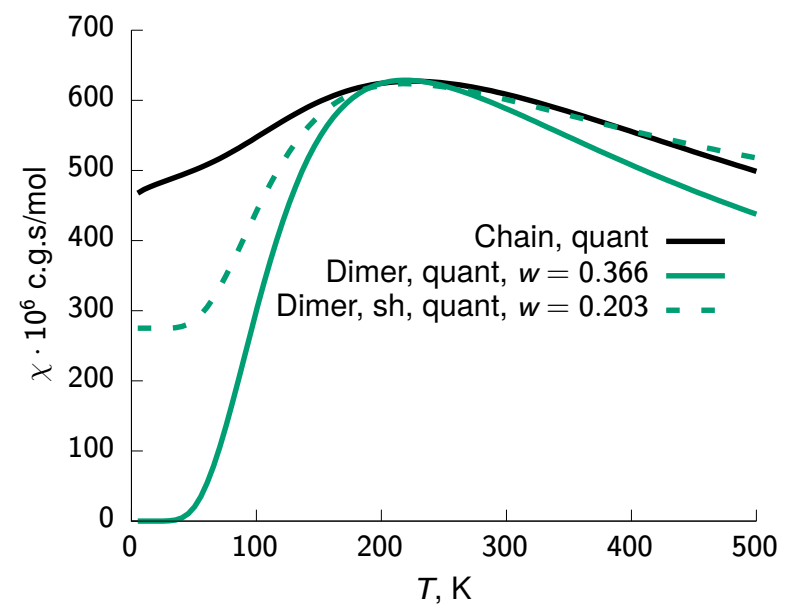

Figure S4: Comparison of susceptibility of the quantum infinite chain and dimer models. Two graphs for the dimer are shown: solid green is the susceptibility of the dimer model that has been scaled by the factor of 0.366 ; the dashed line is the susceptibility of the dimer model that has been scaled by the factor 0.201 and shifted to match the susceptibility of the infinite chain model. The weight 0.201 has been used in the Ref. 2. The shifted and scaled dimer susceptibility represents the high-temperature region well, but leads to noticeable differences at lower temperatures. 


\section{Cartesian coordinates}

\$comment

Fractional atom coordinates within a unit cell.

The last column represents the partial charges, used in the work.

\$end

$\begin{array}{rrrrr}\mathrm{Cu} & 0.00000 & 0.00000 & 0.00000 & 0.35465 \\ \mathrm{O} & 0.16200 & 0.16500 & 0.80300 & -0.22450 \\ \mathrm{O} & 0.21500 & 0.15500 & 0.39600 & -0.14845 \\ \mathrm{C} & 0.10900 & 0.09300 & 0.55300 & 0.19560\end{array}$

\section{Relevant Cartesian geometries}

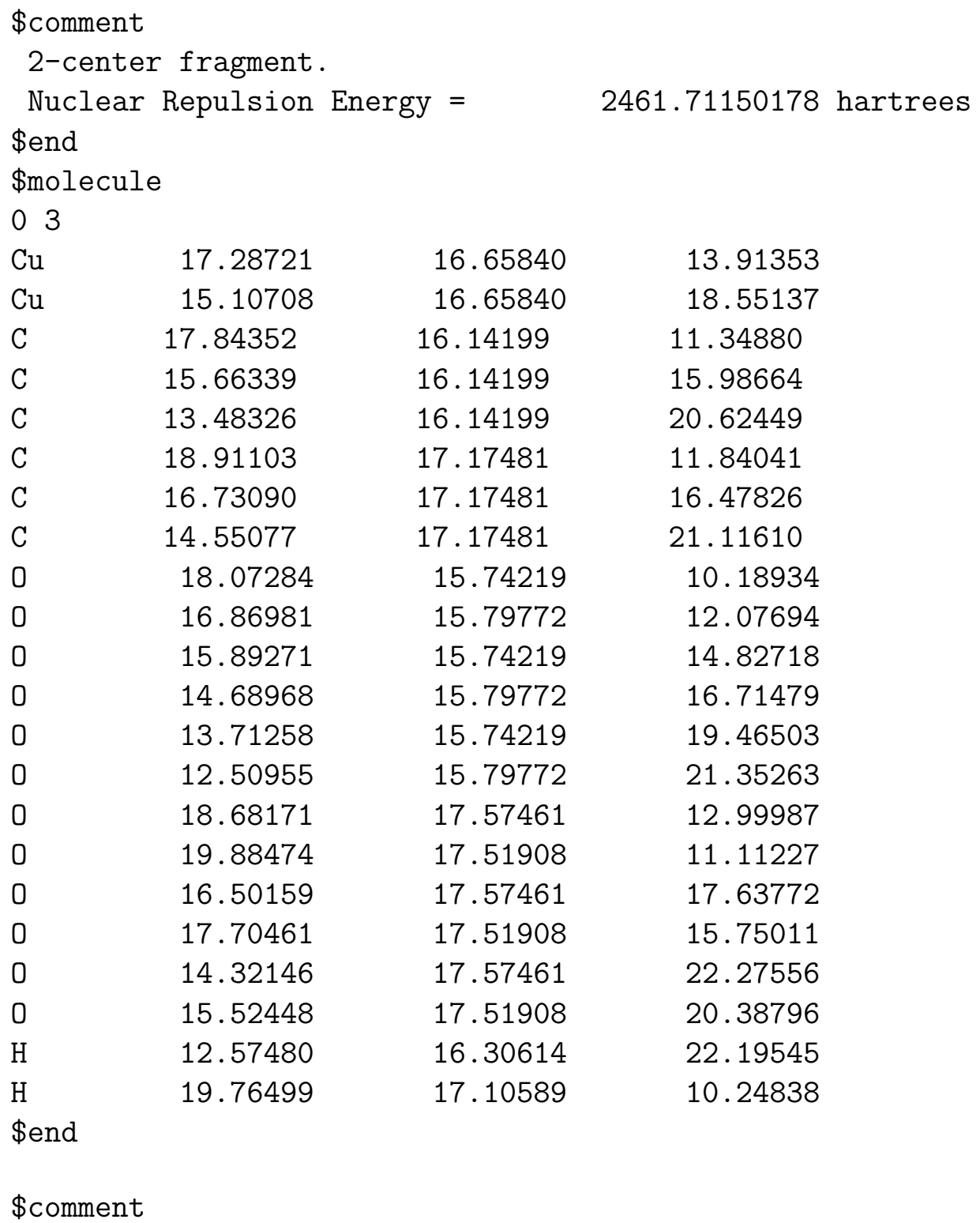




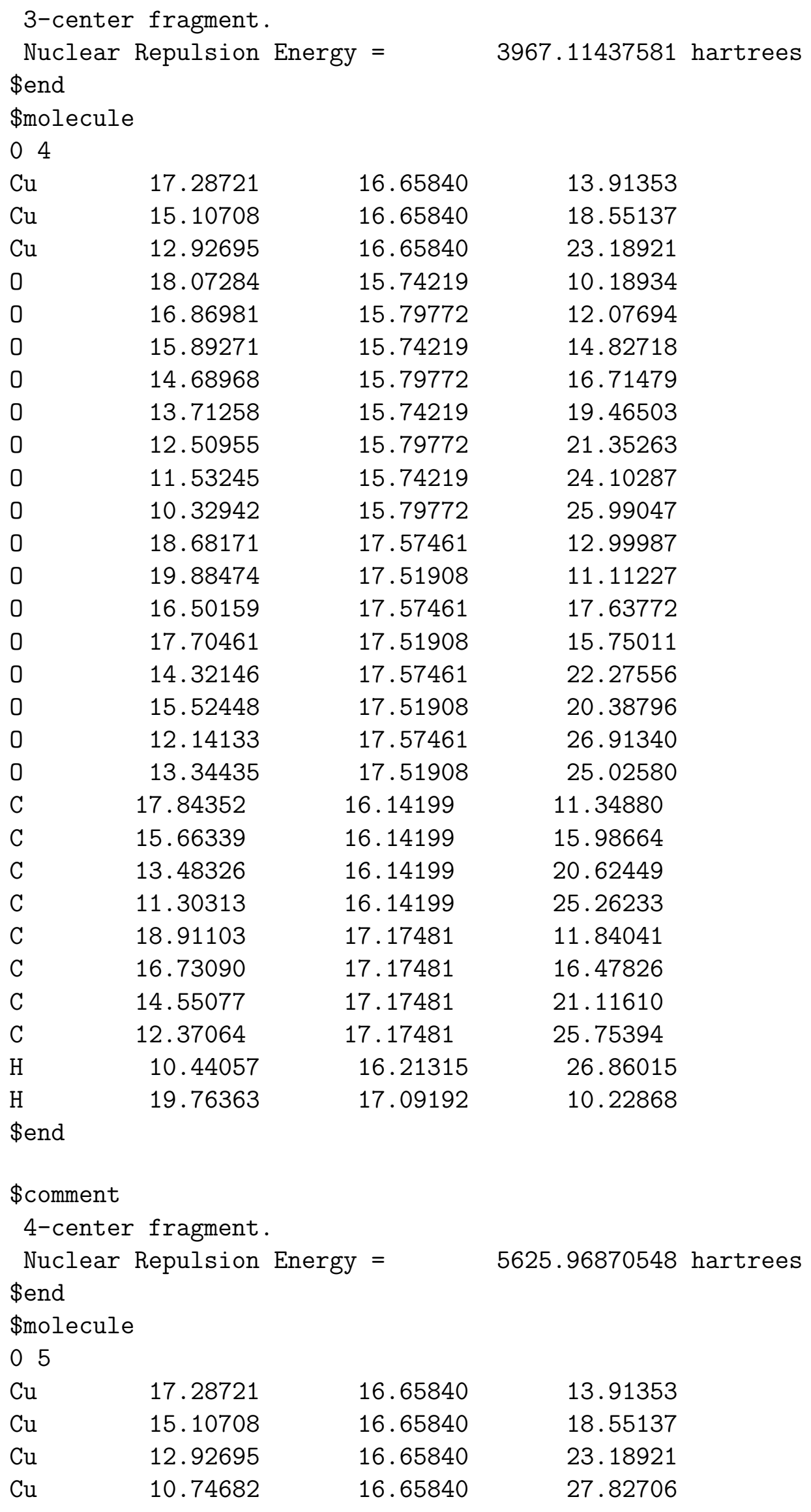




\begin{tabular}{|c|c|c|c|}
\hline 0 & 18.07284 & 15.74219 & 10.18934 \\
\hline 0 & 16.86981 & 15.79772 & 12.07694 \\
\hline 0 & 15.89271 & 15.74219 & 14.82718 \\
\hline 0 & 14.68968 & 15.79772 & 16.71479 \\
\hline 0 & 13.71258 & 15.74219 & 19.46503 \\
\hline U & 12.50955 & 15.79772 & 21.35263 \\
\hline U & 11.53245 & 15.74219 & 24.10287 \\
\hline U & 10.32942 & 15.79772 & 25.99047 \\
\hline U & 9.35232 & 15.74219 & 28.74071 \\
\hline U & 8.14929 & 15.79772 & 30.62831 \\
\hline U & 18.68171 & 17.57461 & 12.99987 \\
\hline U & 19.88474 & 17.51908 & 11.11227 \\
\hline 0 & 16.50159 & 17.57461 & 17.63772 \\
\hline U & 17.70461 & 17.51908 & 15.75011 \\
\hline 0 & 14.32146 & 17.57461 & 22.27556 \\
\hline U & 15.52448 & 17.51908 & 20.38796 \\
\hline & 12.14133 & 17.57461 & 26.91340 \\
\hline 0 & 13.34435 & 17.51908 & 25.02580 \\
\hline & 9.96120 & 17.57461 & 31.55124 \\
\hline 0 & 11.16422 & 17.51908 & 29.66364 \\
\hline C & 17.84352 & 16.14199 & 11.34880 \\
\hline$\checkmark$ & 15.66339 & 16.14199 & 15.98664 \\
\hline$C$ & 13.48326 & 16.14199 & 20.62449 \\
\hline C & 11.30313 & 16.14199 & 25.26233 \\
\hline C & 9.12300 & 16.14199 & 29.90017 \\
\hline $\mathrm{C}$ & 18.91103 & 17.17481 & 11.84041 \\
\hline U & 16.73090 & 17.17481 & 16.47826 \\
\hline C & 14.55077 & 17.17481 & 21.11610 \\
\hline$C$ & 12.37064 & 17.17481 & 25.75394 \\
\hline C & 10.19051 & 17.17481 & 30.39178 \\
\hline $\mathrm{H}$ & 8.22335 & 16.31273 & 31.45165 \\
\hline $\mathrm{H}$ & 19.74448 & 17.10907 & 10.23780 \\
\hline
\end{tabular}




\section{References}

[1] B. N. Figgis and D. J. Martin, The magnetic properties and structure of the cupric $\alpha, \omega-$ dicarboxylates, Inorg. Chem. 5, 100 (1966).

[2] A. N. Christensen, B. Lebech, N. H. Andersen, and J.-C. Grivel, The crystal structure of paramagnetic copper(II) oxalate $\left(\mathrm{CuC}_{2} \mathrm{O}_{4}\right)$ : formation and thermal decomposition of randomly stacked anisotropic nano-sized crystallites, Dalton Trans. 43, 16754 (2014). 